\title{
Diversity of Cis-elements in response to dioxin in human
}

\author{
Evgenia Oschepkova \\ ICG SB RAS, Novosibirsk, Russia \\ nzhenia@bionet.nsc.ru
}

\author{
Yana Sizentsova \\ ICG SB RAS, Novosibirsk, Russia \\ sizentsova.yans@gmail.com
}

\author{
Viktoriya Mironova \\ ICG SB RAS, Novosibirsk, Russia \\ NSU, Novosibirsk, Russia \\ victoria.v.mironova@gmail.com
}

\begin{abstract}
In this work we studied the potential transcriptional regulators which may play role under the action of one of the most dangerous anthropogenic poisons - dioxins. Using limma, edgeR, MetaR, HOMER, Tomtom and MCOT bioinformatics tools for microarray and RNA-seq data analyzing identification of new possible cis-elements involved in the response of cells to dioxins was carried out.
\end{abstract}

Keywords - dioxin, AhR, transcription regulation, ciselements, meta-analysis.

Motivation and aim

\section{Motivation}

Dioxins, widespread environmental contaminants, cause disturbances in the functioning of the immune, endocrine, and reproductive systems; their exposure leads to developmental pathologies in embrios and newborns (in particular cleft palate), cause a decrease in IQ [1-3]. The cellular receptor to which dioxins are bind (also being the main transcriptional regulator) is known. However, the effect of dioxins has a wide range of changes in the organism, the expression of hundreds of genes are changes. The mechanisms underlying this are not clear.

Aim

Our goal was to identify potential transcription factors (TF) that may be involved in the response of cells to TCDD (2,3,7,8-tetrachlorodibenzo-p-dioxin), in addition to those whose functioning has already been shown in numerous studies. Using microarray and RNA-seq data, as well as bioinformatics tools, we searched for potential cis-elements in genes that systematically changing expression in response to dioxin.

\section{Methods}

Package limma [4] for microarray and edgeR [5] for RNAseq datasets were used to distinguish differentially expressed genes (DEGs), which are changing their expression in response to dioxin. Two types of samples were used: TCDDtreated cell cultures and control. False discovery rate (FDR) $\leqslant 0.2$ and fold-change $>1.3$. Microarray and RNA-seq datasets were taken from GEO database. To identify overreprsented potential cis-elements in promoters of DEGs the R package MetaRE [6] and HOMER v4.10 [7] were used. For this the upstream regions [-1500:+1] of 19815 Homo sapiens genes were taken from GENCODE Release v27. Tomtom motif comparison tool [8] was used to comparing overrepresented sequences founded by MetaRE with known transcription factor (TF) cis-elements (HOCOMOCO v11 core database with using the euclidean distance). The hits with an E-value $<0.05$ were considered as significant matches. MCOT Toolbox [9] was used to identify composite elements, functioning together with aryl hydrocarbon receptor (AhR).

\section{Results}

As a result of a meta-analysis of the upstream regions of DEGs that change expression in response to TCDD, hundreds of frequently occurring short (from 5 to 8 nucleotides) sequences were found. Using the Tomtom software package, an analysis of the correspondence of these sequences to the binding sites of known transcription factors was carried out. According to the generally accepted concept of the TCDD action on cells, the main mediator is AhR, which regulates genes by binding to the dioxin-responsive elements (DRE) in DNA. However, AhR binding sites were far from first among the other overrepresented potential cis-elements. The most frequently occurred transcription factors in results of analysis are member of KLF ad SP families. MCOT expectedly shown the presence of AhR-ARNT composite elements. Besides this composite elements with other TFs were found, in particular with KLF, ZNF and E2F families members.

\section{ACKNOWLEDGMENT}

Supported by RFBR (18-04-01130) and by the Complex Program for Fundamental Research of SB RAS.

\section{REFERENCES}

[1] Yoshioka W., Tohyama C. (2019) Mechanisms of Developmental Toxicity of Dioxins and Related Compounds. Int J Mol Sci. 20(3). pii: E617.

[2] Lundqvist C., Zuurbier M., Leijs M., Johansson C., Ceccatelli S., Saunders M., Schoeters G., ten Tusscher G., Koppe J.G. (2006) The effects of PCBs and dioxins on child health. Acta Paediatr Suppl. 95:55-64.

[3] Wesselink A., Warner M., Samuels S., Parigi A., Brambilla P., Mocarelli P., Eskenazi B. (2014) Maternal dioxin exposure and pregnancy outcomes over 30 years of follow-up in Seveso. Environ Int. 63:143-8.

[4] Ritchie M.E., Phipson B., Wu D., Hu Y., Law C.W., Shi W., Smyth G.K. (2015) limma powers differential expression analyses for RNAsequencing and microarray studies. Nucleic Acids Res. 43:e47.

[5] Robinson M.D., McCarthy D.J., Smyth G.K. (2010) edgeR: a Bioconductor package for differential expression analysis of digital gene expression data. Bioinformatics. 26:139-40.

[6] Cherenkov P., Novikova D., Omelyanchuk N., Levitsky V., Grosse I., Weijers D., Mironova V. (2018) Diversity of cis-regulatory elements associated with auxin response in Arabidopsis thaliana. J Exp Bot. 69(2):329-339.

[7] Heinz S., Benner C., Spann N., Bertolino E., Lin Y.C., Laslo P., Cheng J.X., Murre C., Singh H., Glass C.K. Simple combinations of lineagedetermining transcription factors prime cis-regulatory elements required for macrophage and B cell identities. Mol Cell. 2010;38:57689.

[8] Bailey T.L., Boden M., Buske F.A., Frith M., Grant C.E., Clementi L., Ren J., Li W.W., Noble W.S. (2009) MEME SUITE: tools for motif discovery and searching. Nucleic Acids Res. 37:W202-8.

[9] Levitsky V., Zemlyanskaya E., Oshchepkov D., Podkolodnaya O., Ignatieva E., Grosse I., Mironova V., Merkulova T. (2019) A single ChIP-seq dataset is sufficient for comprehensive analysis of motifs cooccurrence with MCOT package. Nucleic Acids Res. 47(21):e13 